\title{
EEG suppression ratio monitoring in successfully resuscitated out-of-hospital cardiac arrest patients: a novel prognostic marker
}

\author{
S. Bouneb, D. Mesotten, R. Heylen, J. Dens, W. Eertmans, C. De Deyne
}

Department of Medicine and Life Sciences, Hasselt University, Diepenbeek, Belgium Department of Anesthesiology, Intensive Care, Emergency Medicine and Pain Therapy, ZOL Genk, Belgium

\section{INTRODUCTION}

This study investigates the prognostic role of the suppression ratio (SR) in EEG in out-of-hospital cardiac arrest (OHCA) patients admitted to the ICU.

\section{METHODS}

- After admission to the ICU, BIS monitoring was initiated in 77 successfully resuscitated OHCA patients using BIS VISTA ${ }^{\text {TM }}$ device (Aspect Medical Systems, Inc. Norwood, USA).

- Targeted temperature management (TTM) at $33^{\circ} \mathrm{C}$ was used (i.e. 24 hours), followed by a rewarming phase of 12 hours.
- Mean SR values were calculated per hour and used for further data analysis.

- At 180 days post cardiac, neurological outcome was defined using the cerebral performance category where a CPC 1-2 was defined as good and CPC 3-5 was defined as poor neurological outcome.

\section{RESULTS}

Figure 1. The evolution of $S R$ during $T T M$ at $33^{\circ} \mathrm{C}$ for patients with a good $(N=38)$ and a poor neurological outcome $(N=39)$.

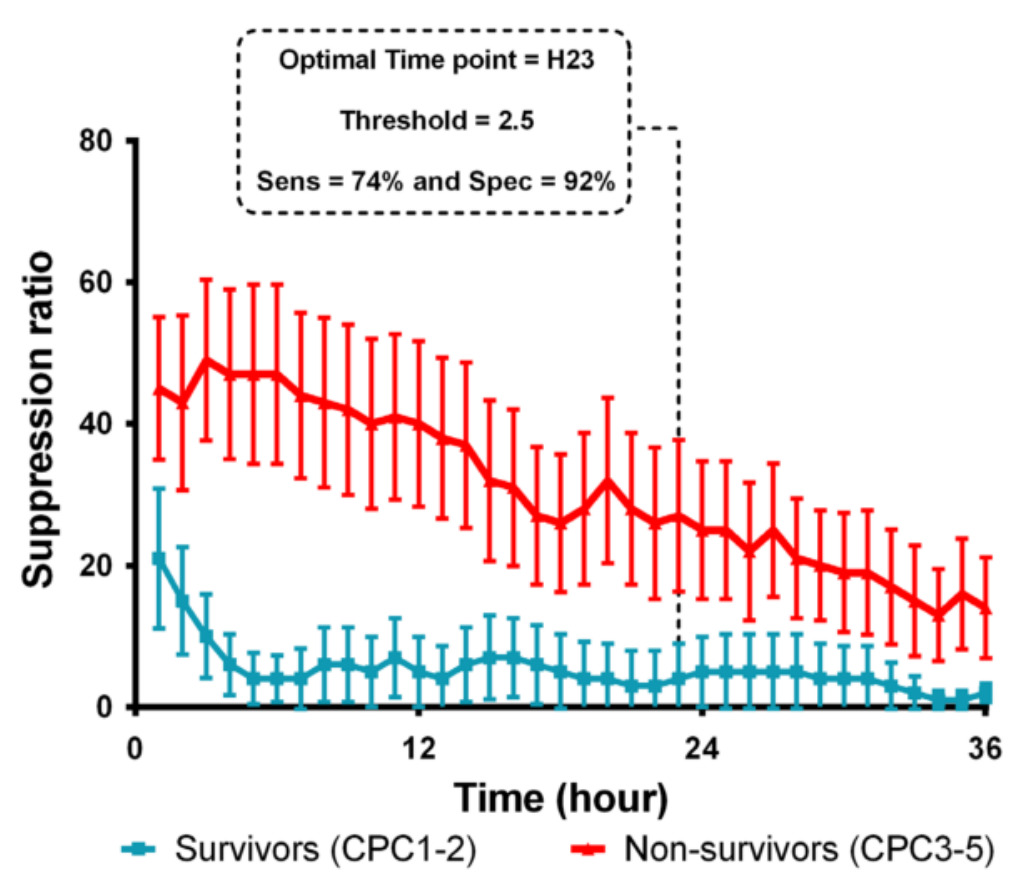

Figure 2. Forest plot analysis at hour 23.

Forest plot SR

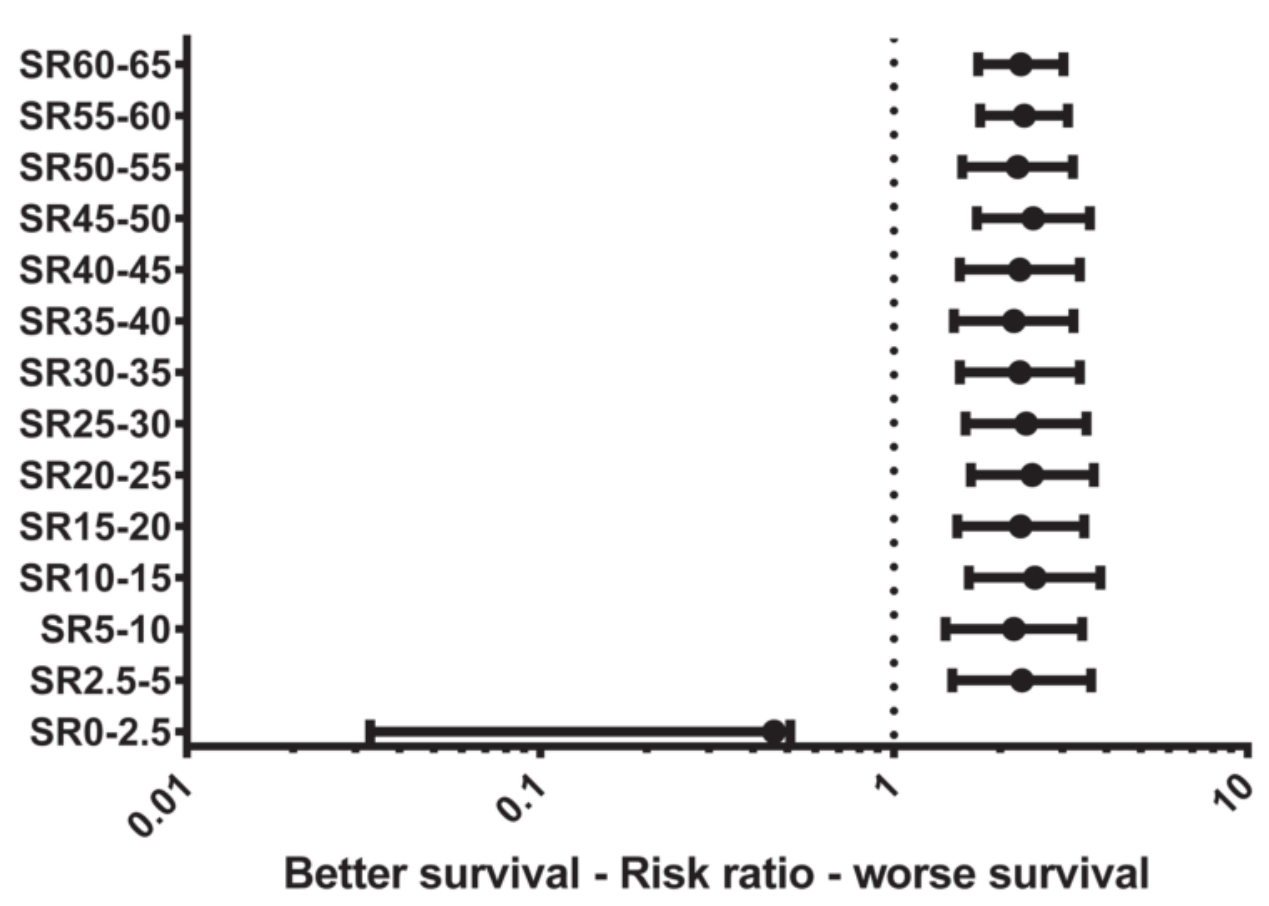

Figure 3. Survival analysis.

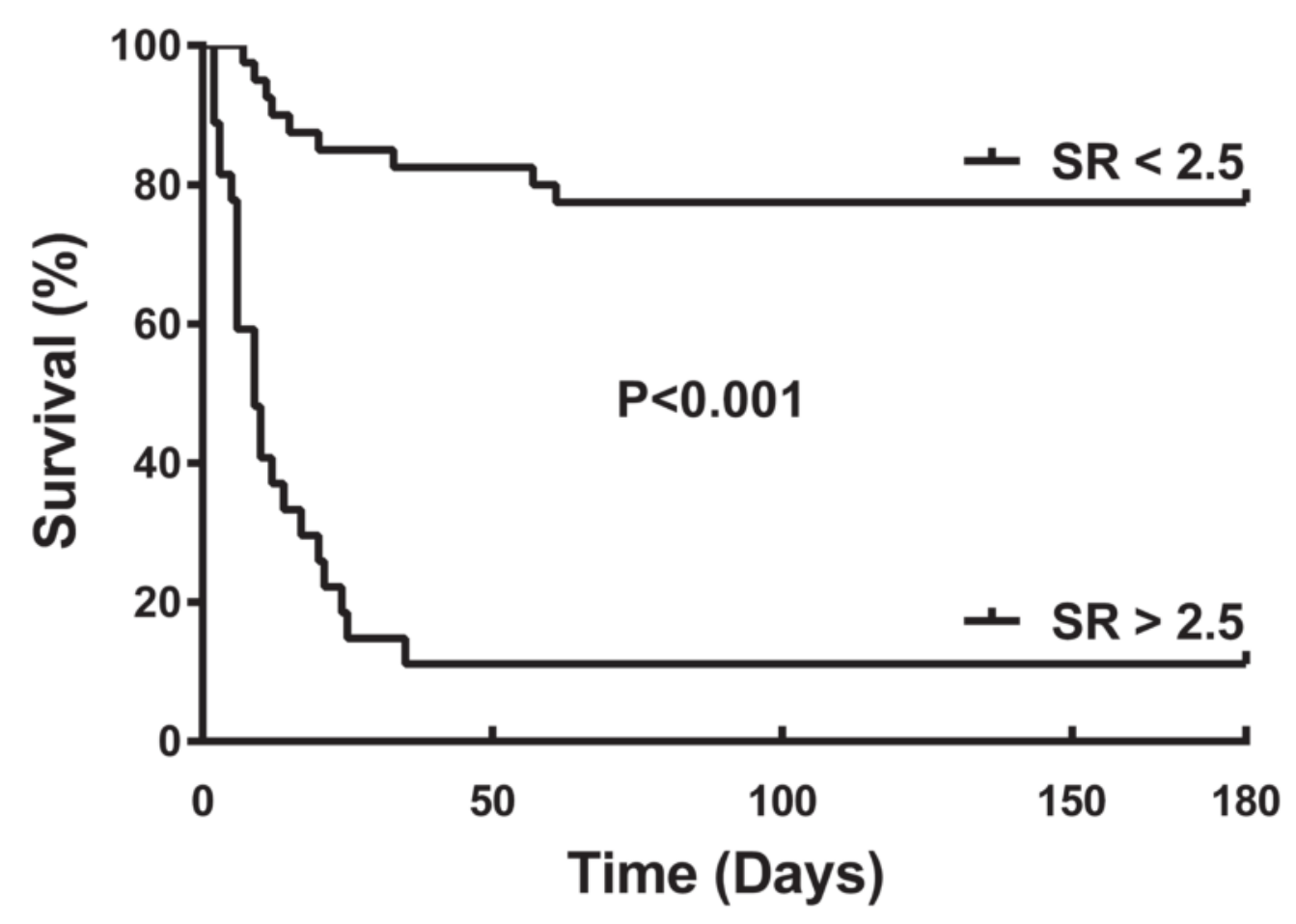

\begin{tabular}{r|c|c|r} 
Ranges & $R R$ & $95 \% \mathrm{Cl}$ & \multicolumn{1}{c}{$P$} \\
\hline $60-65$ & 2.286 & $1.731-3.018$ & 0.008 \\
$55-60$ & 2.333 & $1.754-3.103$ & 0.004 \\
$50-55$ & 2.235 & $1.559-3.204$ & 0.002 \\
$45-50$ & 2.476 & $1.714-3.578$ & $<0.001$ \\
$40-45$ & 2.270 & $1.536-3.354$ & 0.001 \\
$35-40$ & 2.182 & $1.478-3.221$ & 0.002 \\
$30-35$ & 2.270 & $1.536-3.354$ & 0.001 \\
$25-30$ & 2.363 & $1.593-3.503$ & $<0.001$ \\
$20-25$ & 2.461 & $1.650-3.671$ & $<0.001$ \\
$15-20$ & 2.281 & $1.508-3.449$ & 0.001 \\
$10-15$ & 2.500 & $1.630-3.835$ & $<0.001$ \\
$5-10$ & 2.182 & $1.399-3.402$ & 0.001 \\
$2.5-5$ & 2.299 & $1.462-3.614$ & 0.001 \\
$0-2.5$ & 0.458 & $0.033-0.509$ & 0.001
\end{tabular}

\section{CONCLUSIONS}

This prospective, observational study confirmed that mean SR values at hour 23 with a SR value above 2.5 can be used to predict poor neurological outcome.

universiteit $\rightarrow$ hasselt KNOWLEDGEINACTION 